\title{
Functional and structural connectivity of the executive control network in college binge drinkers
}

\author{
Sónia S. Sousa ${ }^{\mathrm{a}, *}$, Adriana Sampaio ${ }^{\mathrm{a}}$, Paulo Marques $^{\mathrm{b}, \mathrm{c}}$, Eduardo López-Caneda ${ }^{\mathrm{a}}$, \\ Óscar F. Gonçalves ${ }^{\mathrm{a}, \mathrm{d}, \mathrm{e}}$, Alberto Crego ${ }^{\mathrm{a}}$ \\ ${ }^{a}$ Psychological Neuroscience Lab, CIPsi, School of Psychology, University of Minho, Campus Gualtar, 4710-057 Braga, Portugal \\ ${ }^{\mathrm{b}}$ Life and Health Sciences Research Institute (ICVS), School of Health Sciences, University of Minho, Campus Gualtar, 4710-057 Braga, Portugal \\ ' ICVS/3B's - PT Government Associate Laboratory, Braga/Guimarães, Portugal \\ ${ }^{\mathrm{d}}$ Spaulding Neuromodulation Center, Department of Physical Medicine and Rehabilitation, Spaulding Rehabilitation Hospital, Massachusetts General Hospital. Harvard \\ Medical School, Charlestown campus: 79/96 13th Street, Charlestown, MA 02129, USA \\ ${ }^{\mathrm{e}}$ Department of Applied Psychology, Bouvé College of Health Sciences, Northeastern University, 404 International Village, Boston, MA 02115, USA
}

\section{H I G H L I G H T S}

- Increased rsFC in the LECN middle frontal gyrus of college BDs.

- No between group's differences were observed in the RECN.

- ECN structural connectivity analysis revealed no between group's differences.

- LECN rsFC was positively associated to the frequency of BD episodes.

- Intermittent acute alcohol consumption during youth may disrupt rsFC of the LECN.

\section{A R T I C L E I N F O}

\section{Keywords:}

Binge drinking

Executive control network

Resting-state fMRI

DTI

College-students

\begin{abstract}
A B S T R A C T
Binge Drinking (BD) is a pattern of excessive alcohol consumption highly prevalent among college students, and has been associated with structural and functional alterations of brain networks. Recent advances in the restingstate connectivity analysis have boosted the research of the network-level connectivity disturbances associated with many psychiatric and neurological disorders, including addiction. Accordingly, atypical functional connectivity patterns in resting-state networks such as the Executive Control Network (ECN) have been found in substance users and alcohol-dependent individuals. In this study, we assessed for the first time the ECN functional and structural connectivity in a group of 34 college students, 20 (10 women) binge drinkers (BDs) in comparison with a group of 14 (8 women) alcohol abstinent controls (AACs).

Overall, our findings documented increased resting-state functional connectivity (rsFC) in the BDs left middle frontal cortex of the left ECN in comparison to the AACs, while no structural connectivity differences were observed between groups. Pearson correlations revealed a positive association between the left middle frontal gyrus rsFC and the frequency of BD episodes per month, in the BD group.

These findings suggest that maintaining a pattern of acute and intermittent alcohol consumption during important stages of brain development, as the transition from adolescence to adulthood, is associated with impaired ECN rsFC despite no group differences being yet noticed in the ECN structural connectivity.
\end{abstract}

\section{Introduction}

According to the National Institute on Alcohol Abuse and Alcoholism, repeated episodes of excessive alcohol consumption, at least five unit drinks for men and four for women, in approximately $2 \mathrm{~h}$, followed by periods of abstinence characterize binge drinking (BD).
This highly prevalent pattern of alcohol consumption among adolescents and young adults has been associated with significant detrimental health, social and academic consequences (NIAAA, 2015). BD typically emerges during late adolescence and especially college students are the ones who engage more in binge episodes (Courtney \& Polich, 2009; NIAAA, 2015).

\footnotetext{
* Corresponding author.

E-mail address: soniamachado@psi.uminho.pt (S.S. Sousa).
} 
Alcohol misuse, including adolescent $\mathrm{BD}$, has been related to the youth tendency to underestimate risk, high impulsiveness and difficulties in behavioral regulation (Bari \& Robbins, 2013; Crews \& Boettiger, 2009). These particular characteristics of the youth behavior seem to be associated with the structural and functional immaturity of the prefrontal cortex (PFC), a region still undergoing deep neurodevelopmental changes during this period (Bava \& Tapert, 2010; Casey, Jones, \& Somerville, 2011). Additionally, recent research has shown that among other cognitive impairments, inhibitory control seems to be one of the most affected cognitive processes in college BDs (for a recent review, see Carbia, Lopez-Caneda, Corral, \& Cadaveira, 2018). As previously reported, this cognitive function relies upon fronto-striatal circuitry integrity (Aron et al., 2007; Feil et al., 2010), which was recently shown as being altered in college BDs (Sousa, Sampaio, Marques, Goncalves, \& Crego, 2017).

Analysis of resting-state networks (RSNs) has received considerable attention over the last decade due to its valuable contributions in exploring the network-level disruptions in multiple psychiatric conditions (Menon, 2011; van den Heuvel \& Hulshoff Pol, 2010), including addictive disorders (Pariyadath, Gowin, \& Stein, 2016; Sutherland, McHugh, Pariyadath, \& Stein, 2012). In this sense, several studies addressing the brain functional networks point to abnormal resting-state functional connectivity (rsFC) patterns within and between nodes of RSNs, namely the Default Mode Network (DMN), the Executive Control Network (ECN) and the Salience Network (SN) in individuals with alcohol and other drugs abuse (Camchong, Stenger, \& Fein, 2013; Dupuy \& Chanraud, 2016; Fedota \& Stein, 2015; Jansen, van Wingen, van den Brink, \& Goudriaan, 2015; Ma, Steinberg, Moeller, Johns, \& Narayana, 2015; Muller-Oehring et al., 2015; Sutherland et al., 2015; Vergara, Liu, Claus, Hutchison, \& Calhoun, 2017; Zhu, Cortes, Mathur, Tomasi, \& Momenan, 2015). Additionally, recent reports also noticed altered rsFC in the Ventral Attentional Network (VAN), and between the amygdala and orbitofrontal cortex (OFC), in adult BDs (age range: 18-40) (Crane, Gorka, Phan, \& Childs, 2018; Herman, Critchley, \& Duka, 2018). Specifically, decreased within-network rsFC was observed in the right supramarginal gyrus of the VAN, and in the right seed-based amygdalaOFC rsFC, further associated with higher scores of alcohol use (Crane et al., 2018; Herman et al., 2018).

Likewise, structural connectivity analyses using diffusion magnetic resonance imaging (dMRI) have recently been considered a complementary method to further explore the RSN's structural connectivity. White matter bundles are responsible for the neurotransmission between anatomically separated brain regions that show temporal dependency in a task-negative state (van den Heuvel \& Hulshoff Pol, 2010). Therefore, the combination of both measures -functional MRI (fMRI) and dMRI- seems to enable a more comprehensive understanding of the effects of alcohol abuse on brain networks (Schulte, Müller-Oehring, Rohlfing, Pfefferbaum, \& Sullivan, 2010; Schulte, Müller-Oehring, Sullivan, \& Pfefferbaum, 2012).

Despite its well-documented potential for detecting network functional connectivity disturbances in chronic alcoholics, rsFC analysis has been virtually unaddressed in the young BD population. Indeed, to the best of our knowledge, only a few studies have directly assessed -applying magnetoencephalograpy (MEG) and electroencephalography (EEG) techniques- the effects of $\mathrm{BD}$ on the brain activity at rest in young BDs (Affan et al., 2018; Correas et al., 2015, 2016; Courtney \& Polich, 2010; Lopez-Caneda et al., 2017). However, the only study combining structural and functional connectivity analysis in this population was a seed-based MEG (Correas et al., 2016, which revealed enhanced DMN rsFC in college BDs as compared to controls. This effect further increased after two years maintaining the BD behavior, whereas no structural connectivity differences were observed between groups (Correas et al., 2016). Additionally, EEG and MEG studies reported that BDs exhibited increased functional connectivity in the theta band of a fronto-parietal network (Correas et al., 2015) as well as augmented theta and beta power over frontal regions, which was further associated with several drinking variables (such as the number of $\mathrm{BD}$ episodes, the maximum number of drinks in $24 \mathrm{~h}$, etc.) (Affan et al., 2018).

The ECN is a fronto-parietal network that includes nodes in the dorsolateral prefrontal cortex (DLPFC) and the lateral parietal cortex, being apparently engaged in high-level cognitive functions such as working memory, cognitive control and goal-directed behavior (Menon, 2011; Niendam et al., 2012; Seeley et al., 2007). Its role in key cognitive processes involved in the regulation of substance use behaviors makes the study of this network particularly relevant to the field of alcohol abuse. In accordance, resting-state functional synchrony abnormalities within the nodes of the ECN have been observed in alcohol-dependent subjects (e.g. Jansen et al., 2015; Weiland et al., 2014). However, the connectivity strength of such abnormal rsFC is not yet clearly determined. While some of the studies observed weaker within-network rsFC in the ECN of alcoholics and adult BDs as compared to healthy subjects (Müller-Oehring et al., 2015; Weiland et al., 2014), others reported increased intrinsic ECN rsFC in these individuals (Jansen et al., 2015; Krmpotich et al., 2013; Zhu et al., 2015).

Therefore, in this study we addressed, for the first time, the study of the ECN functional and structural connectivity in young BDs. Based on the literature, we hypothesized that -compared with alcohol abstinent controls (AACs)- 1) BDs will show abnormal rsFC; and 2) disrupted structural organization and axonal branching -as indexed by fractional anisotropy (FA), mean diffusivity (MD), axial diffusivity (AD), and radial diffusivity (RD)- within the ECN. The hypothesized alterations may eventually be more prominent in the DLPFC considering the role of these regions in inhibitory control processes.

\section{Method}

\subsection{Participants}

Thirty-four college students within the age range 18 to 23 years old, 20 BDs ( 10 women; mean age: 20 years) and 14 AACs ( 8 women; mean age: 20 years), were included in this study. Individuals with a minimum alcohol consumption of five drinks (four for women) during a short time interval $(2 \mathrm{~h})$, at least once per month for the past 10 months, were assigned to the BD group. Participants assigned to the AAC group were completely alcohol abstinent, i.e., do not drink alcohol at all, neither now nor in the past.

Before the MRI assessment, all the participants were requested to avoid BD episodes during the three preceding days, consuming illicit drugs and alcohol twelve hours before the scanning, and restrain smoking and tea or coffee consumption for at least $3 \mathrm{~h}$ in advance. Participants were informed about the research procedure, gave written informed consent and received a financial stipend. The research was conducted in accordance to the ethical principles for medical research involving human subjects of the World Medical Association (WMA) present in the Declaration of Helsinki (WMA, 2013). Please see Table 1 for more details about participants.

Exclusion criteria for both groups were defined as the following: be left-handed; scores 20 in the AUDIT; GSI 90 or scoring in at least 2 symptomatic dimensions of the SCL- 90-R; uncorrected sensory deficits; personal history of psychopathology, traumatic brain injury or neurological disorder; regular (i.e., on a weekly basis) consumption of cannabis, personal history of regular or occasional use of other illicit drugs or psychoactive substances; Alcohol Use Disorder; personal and/or family history of any neurological or DSM-IV axis I disorder in first-degree relatives, family history of alcoholism in first-degree relatives; and magnetic resonance imaging (MRI) contraindications.

\subsection{MRI acquisition}

The resting-state fMRI images were acquired using a blood oxygen level dependent (BOLD) sensitive echo-planar imaging (EPI) with the following parameters: 39 interleaved axial slices; TR $=2000 \mathrm{~ms}$; 
Table 1

Demographic and behavioral data for binge drinkers and alcohol abstinent controls.

\begin{tabular}{|c|c|c|c|}
\hline & $\begin{array}{l}\text { BD } \\
N=20 \\
\text { Mean (SD) }\end{array}$ & $\begin{array}{l}\text { AAC } \\
N=14 \\
\text { Mean (SD) }\end{array}$ & $\mathrm{t}(32)$ \\
\hline$\%$ Male & $50 \%$ & $42.9 \%$ & \\
\hline$\%$ Female & $50 \%$ & $57.1 \%$ & \\
\hline$\%$ Caucasian & 100 & 100 & \\
\hline Age & $20.45(1.60)$ & $20.86(1.75)$ & 0.70 \\
\hline Age of onset of BD & $17.45(1.08)$ & - & \\
\hline AUDIT (total score) & $11.20 \pm 3.25$ & $0.62 \pm 1.20$ & $13.43_{* * *}$ \\
\hline Number of times of BD per month & $3.57 \pm 1.87$ & 0 & $8.54_{\text {水水米 }}$ \\
\hline Number of months with BD pattern & $35.90 \pm 14.03$ & 0 & $11.44_{* * *}$ \\
\hline $\begin{array}{l}\text { Grams of alcohol consumed per } \\
\text { week }\end{array}$ & $151 \pm 44.27$ & 0 & $14.78_{* * *}$ \\
\hline $\begin{array}{l}\text { Speed of drinking (gr/h during BD } \\
\text { episodes) }\end{array}$ & $34.50 \pm 8.26$ & 0 & $18.69_{* * *}$ \\
\hline $\begin{array}{l}\text { Percentage of times getting drunk } \\
\text { when drinking }\end{array}$ & $43.25 \pm 20.41$ & 0 & $9.48_{\text {水水水 }}$ \\
\hline Tobacco Smokers & $7(4$ O) & 0 & \\
\hline Occasional users of Cannabis & $2(2$ Q $)$ & 0 & \\
\hline
\end{tabular}

AUDIT: Alcohol Use Disorders Identification Test; BD: binge drinking; AAC: alcohol-abstinent control; SD: standard deviation.

All $p$-values reported are for 2-tailed independent samples $t$-tests (BD-AAC). **** $P \leq .001$.

$\mathrm{TE}=29 \mathrm{~ms} ; \mathrm{FA}=90^{\circ}$; slice thickness $=3$; in-plane resolution $=3 \times 3$ $\mathrm{mm}^{2} ; \mathrm{FoV}=222 \mathrm{~mm}$ and 210 volumes. The Diffusion Weighted Imaging (DWI) was performed using a spin-echo echo-planar imaging (SE-EPI) sequence: $\quad \mathrm{TR}=9300 \mathrm{~ms} ; \quad \mathrm{TE}=93 \mathrm{~ms} ; \quad \mathrm{FoV}=204.8 \times$ $204.8 \mathrm{~mm}$; acquisition matrix $=128 \times 128$; $652 \mathrm{~mm}$ axial slices with no gap; 64 non-collinear gradient directions with $\mathrm{b}=700 \mathrm{~s} / \mathrm{mm}^{2}$; and one $\mathrm{b}=0 \mathrm{~s} / \mathrm{mm}^{2}$ acquisition. MP-RAGE acquisition parameters were: repetition time $(\mathrm{TR})=2700 \mathrm{~ms}$; echo time $(\mathrm{TE})=2.33 \mathrm{~ms}$; slice thickness $=0.8 \mathrm{~mm}$; in-plane resolution $=1 \times 1 \mathrm{~mm}^{2}$; flip angle $(\mathrm{FA})=7^{\circ}$; field of view $(\mathrm{FoV})=256 \mathrm{~mm}$. Before data pre-processing, the raw MRI and DWI acquisitions were visually inspected to discard the possibility of any brain lesions and/or critical head motion or artifacts that could compromise the analysis.

\subsection{Resting-state fMRI preprocessing}

The preprocessing procedures were applied using FMRIB Software Library (FSL v5.08; http://fsl.fmrib.ox.ac.uk/fsl/) tools. The pipeline was as following. The first $10 \mathrm{~s}$ of acquisition (5 volumes) were discarded due to participant's adjustment to the scanner noise and to achieve signal stabilization. Slice timing was performed using as reference the first acquired slice, followed by head motion correction using MCFLIRT tool (Jenkinson et al., 2002). Motion scrubbing was performed using the fsl_motion_outliers tool to detect timepoints in the fMRI dataset that might have been corrupted by large motion (Power, Barnes, Snyder, Schlaggar, \& Petersen, 2012). Time-points identified as outliers were removed in the dual regression stage of the Independent Component Analysis (ICA). No subject presented $>3 \mathrm{~mm}$ in translation or $3^{\circ}$ in rotation and no subject ended with $<5 \mathrm{~min}$ of useable functional acquisition after accounting for motion outliers. Non-linear spatial normalization to Montreal Neurological Institute (MNI) standard space was then performed through: i) skull stripping of the mean functional image; ii) rigid-body registration of the mean functional image to the skull stripped structural scan; iii) affine registration of the structural scan to the MNI T1 template; iv) non-linear registration of the structural scan to the MNI T1 template using the affine transformation estimated previously as the initial alignment; v) non-linear transformation of the functional acquisition to MNI standard space trough the sequential application of the rigid-body transformation and the nonlinear warp followed and resampling to $2 \times 2 \times 2 \mathrm{~mm}^{3}$ voxel size. Finally, spatial smoothing was performed with a $8 \mathrm{~mm}$ Full-Width at Half Maximum (FWHM) Gaussian kernel followed by band-pass temporal filtering $(0.01-0.08 \mathrm{~Hz})$.

\subsection{Independent component analysis}

Probabilistic Independent Component Analysis (PICA) is a data driven analysis that isolates components or non-overlapping spatial maps corresponding to regions evidencing coherent time-courses (Beckmann \& Smith, 2004). PICA was performed with Multivariate Exploratory Linear Optimized Decomposition into Independent Components (MELODIC), distributed with FSL (Beckmann \& Smith, 2004), as follows: i) participants' preprocessed acquisitions were temporally concatenated into a single 4D dataset; ii) group-wise spatial maps corresponding mainly to RSNs were estimated; iii) automatized estimation of the number of independent components was conducted; iv) subject-specific components were estimated with the dual regression procedure. Confounding factors, namely motion parameters (rotation and translation estimates), motion outliers and mean white matter and cerebrospinal fluid signals were included in the second regression stage of the dual regression procedure.

A group of functionally linked components during resting-state emerged. Two RSNs of interest were identified -the left and right ECNby visual inspection in combination with spatial correlations between the obtained RSNs and RSN templates from the Stanford University Functional Imaging in Neuropsychiatric Disorders (FIND) Lab's publicly available dataset (Shirer, Ryali, Rykhlevskaia, Menon, \& Greicius, 2012). Resting-state ICA derived components with spatial correlation values $r>0.25$ with the correspondent RSN template (i.e. FIND Lab RSNs dataset) were considered significant and corrected for multiple comparisons (Smith et al., 2009; Taylor, Gohel, Di, Walter, \& Biswal, 2012). One component was defined as the left executive control network (LECN) with a correlation of 0.37 with the LECN resting-state functional map, and the other as the right executive control network (RECN) with a correlation of 0.39 with the RECN resting-state functional map. The ECN comprises the superior, middle and inferior frontal gyri, including the DLPFC, the anterior cingulate cortex, and the parietal cortex, plus subcortical regions such as the thalamus, the caudate, the putamen, and cerebellar regions (Niendam et al., 2012).

\subsection{Diffusion tensor imaging (DTI) - image processing and analysis}

Image preprocessing was performed with tools provided by the FMRIB Software Library (FSL v5.0.8; http://fsl. fmrib.ox.ac.uk/fsl/). The analysis pipeline was carried out as follows: i) motion and eddy current corrections using FMRIB's Diffusion Toolbox (FDT); ii) brain extraction tool (BET) was applied to the T1 images, and DWI volumes; iii) tensor fitting and scalar maps computation were performed with the Diffusion Toolkit (DTK) (v0.6.2.2; http://trackvis.org/dtk); iv) After tensor estimation, streamline tractography and scalar maps of FA, AD, $\mathrm{RD}$ and MD were generated; v) label-maps extraction was performed using the LECN and RECN templates made available by the FIND Lab (Shirer et al., 2012); vi) the regions of interest (ROIs) were registered into each subject DTI space using FSL; vii) spatial registration of DTI and ROIs fiber tracking; and viii) estimation of the FA, AD, RD and MD parameters using TrackVis (http://trackvis.org/dtk).

\subsection{DTI - ROIs description}

Four regions of interest were defined for the DTI analyses, based on the RSN templates provided by the FIND Lab (Shirer et al., 2012). Specifically, we use the FIND Lab's LECN and RECN templates, which include all the nodes of the ECN for each hemisphere. In addition we extracted the middle frontal gyrus cluster/node from the FIND Lab's LECN template using the FSL tool cluster (FSL v5.08; http://fsl.fmrib.ox. ac.uk/fsl/). These ECN atlases are freely available at the FIND Lab 
website (http://findlab.stanford.edu/functional_ROIs.html).

\subsection{Statistical analysis}

\subsubsection{Functional connectivity}

Two-way analysis of variance of the LECN and RECN maps was performed, as implemented in the SPM12 (Statistical Parametrical Mapping, version 12, http://www.fil.ion.ucl.ac.uk), to analyze rsFC group differences. Gender and group were included as between subject factors and age as covariate. Contrast maps were set at a primary whole-brain p-threshold $<0.001$ uncorrected. To restrict results to the RSNs of interest, the group difference contrast maps were masked with the Stanford's LECN and RECN templates and FWE correction for multiple comparisons (peak voxel $\mathrm{p}_{\mathrm{FWE}}<0.05$ ) was performed across all voxels within the mask.

Additional analysis of variance were conducted within the LECN entering the diffusion metrics -FA, AD, RD and MD- of both the LECN and the LECN cluster/node -middle frontal gyrus- as covariates.

Pearson correlations (with bootstrap corrections, 5000 iterations and $95 \%$ confidence interval) were performed in the BD group with the aim of analyzing the relationship between the rsFC of the left middle frontal gyrus and alcohol-related measures: months with BD pattern, frequency of $\mathrm{BD}$ episodes per month, grams of alcohol consumed per week, speed of drinking (grams/h during BD episodes), percentage of drunkenness and AUDIT scores.

\subsubsection{Structural connectivity}

A Multivariate analysis of variance was performed using SPSS 20.0 for each ROI -LECN, RECN, and a cluster/node (middle frontal gyrus) of the LECN- with gender and group included as between subject factors and age as covariate. Dependent variables were $\mathrm{FA}, \mathrm{AD}, \mathrm{RD}$ and $\mathrm{MD}$ indexes.

\section{Results}

\subsection{Functional connectivity}

Group differences were observed in the LECN rsFC. Specifically, increased rsFC in one of the nodes of the LECN -the middle frontal gyrus- was observed in the BD group (MNI coordinates: [-36,20,50]; $\mathrm{k}=51 ; \mathrm{Z}=4.22$; peak voxel $\mathrm{p}_{\mathrm{FWE}}=0.019$ ) when compared with the AAC group (see Fig. 1). No group differences were observed in the RECN.

These results remained significant when the diffusion metrics of both the LECN and the left middle frontal gyrus node [(FA, MNI coordinates: $[-36,20,50] ; \mathrm{k}=46 ; \mathrm{Z}=4.10$; peak voxel $\mathrm{p}_{\mathrm{FWE}}=0.030$; FA, MNI coordinates: $[-36,20,50] ; \mathrm{k}=48 ; \mathrm{Z}=4.12$; peak voxel $\left.\mathrm{p}_{\mathrm{FWE}}=0.028\right) ;(\mathrm{AD}, \mathrm{MNI}$ coordinates: $[-36,20,50] ; \mathrm{k}=46 ; \mathrm{Z}=4.11$; peak voxel $\left.\mathrm{p}_{\mathrm{FWE}}=0.029\right)$; $\mathrm{AD}$, MNI coordinates: $[-36,20,50] ; \mathrm{k}=45$; $\mathrm{Z}=4.11$; peak voxel $\left.\mathrm{p}_{\mathrm{FWE}}=0.029\right) ; \quad(\mathrm{RD}, \quad \mathrm{MNI}$ coordinates: $[-36,20,50] ; \mathrm{k}=49 ; \mathrm{Z}=4.14$; peak voxel $\left.\mathrm{p}_{\mathrm{FWE}}=0.026\right) ; \mathrm{RD}$, MNI coordinates: $\quad[-36,20,50] ; \quad \mathrm{k}=50 ; \quad \mathrm{Z}=4.15 ; \quad$ peak voxel $\left.\mathrm{p}_{\mathrm{FWE}}=0.025\right)$; and (MD, MNI coordinates: $[-36,20,50] ; \mathrm{k}=48$; $\mathrm{Z}=4.14$; peak voxel $\mathrm{p}_{\mathrm{FWE}}=0.026$; MNI coordinates: $[-36,20,50]$; $\mathrm{k}=48 ; \mathrm{Z}=4.14$; peak voxel $\left.\left.\mathrm{p}_{\mathrm{FWE}}=0.027\right)\right]$ respectively, were included in the SPM model as covariates.

Pearson correlations (using the $Z$-score transformation) within the BD group revealed positive associations between the left middle frontal gyrus rsFC and the frequency of BD episodes in a month $(r=0.46$ $p=.04$ ), see Fig. 3. No significant correlations were found between the rsFC of the left middle frontal gyrus and the remaining variables.

\subsection{Structural connectivity}

No group differences were observed for the $\mathrm{FA}, \mathrm{AD}, \mathrm{RD}$ and $\mathrm{MD}$ indexes in the LECN, RECN, and the middle frontal gyrus node of the LECN. Fig. 2 displays the FA, AD, RD and MD means by ROI for each group.

\section{Discussion}

The present study assessed both the rsFC and the structural connectivity of the left and right ECN in a preclinical group of college BDs. According to our first hypothesis, the findings revealed that, compared with AACs, BDs displayed increased rsFC in the LECN. More specifically the left middle frontal gyrus emerged as the region showing a higher level of temporal dependency with the remaining areas of the network. No between groups differences were observed in the RECN.

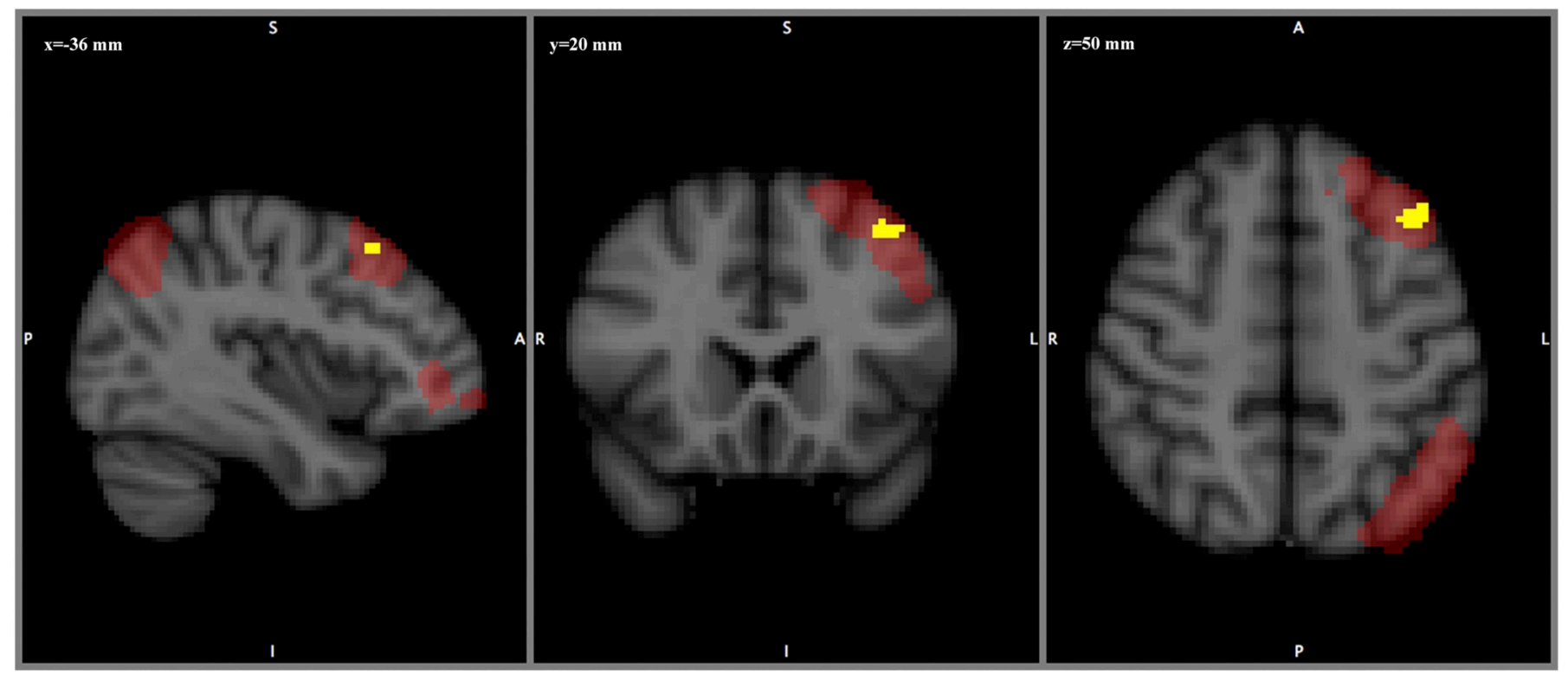

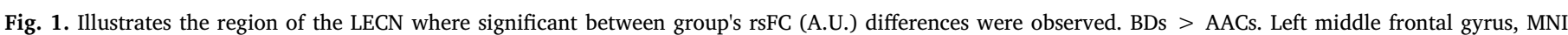

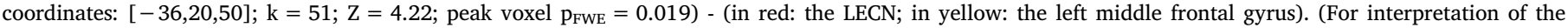
references to colour in this figure legend, the reader is referred to the web version of this article.) 


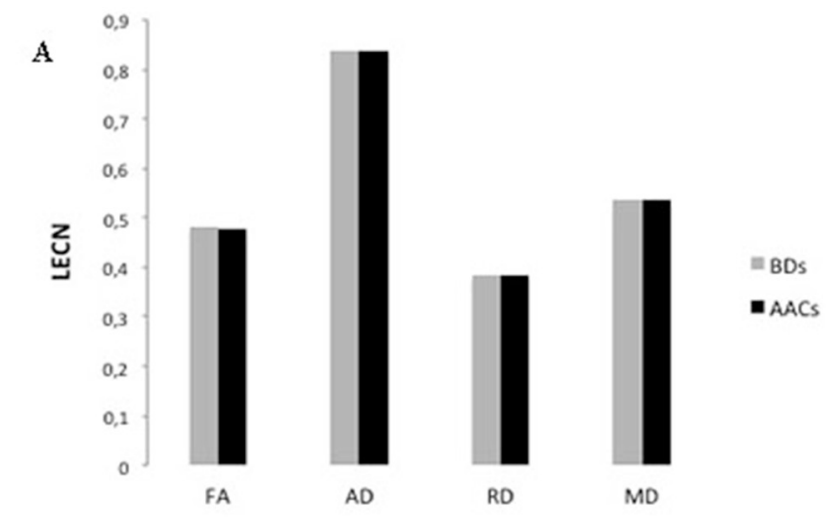

DTI Indexes
B

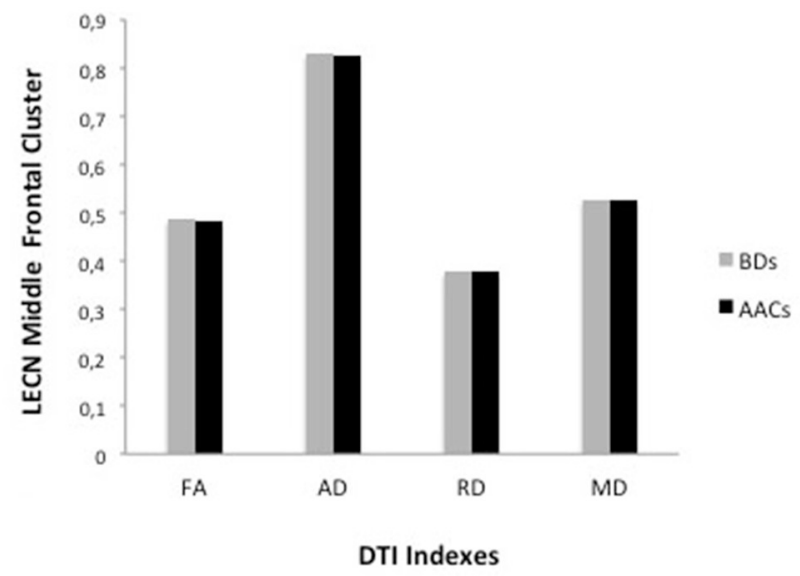

\begin{tabular}{|c|c|c|c|c|c|}
\hline & & FA & $A D$ & RD & MD \\
\hline & & Mean (SD) & Mean (SD) & Mean (SD) & Mean (SD) \\
\hline \multirow[t]{2}{*}{ LECN } & BDs & $0,480(0,014)$ & $0,838(0,019)$ & $0,382(0,011)$ & $0,535(0,012)$ \\
\hline & AACS & $0,479(0,014)$ & $0,836(0,019)$ & $0,383(0,011)$ & $0,534(0,011)$ \\
\hline
\end{tabular}

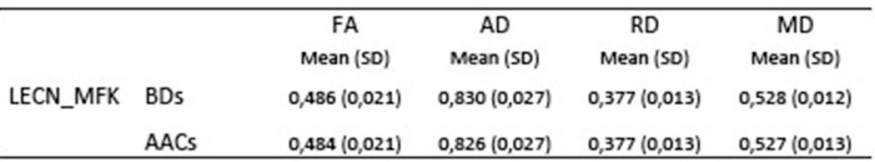

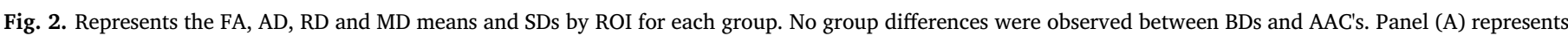

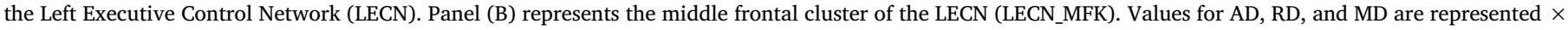

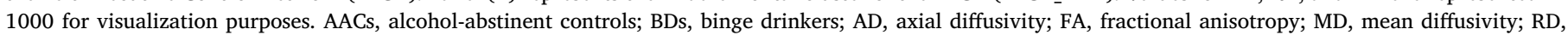
radial diffusivity.

The increased rsFC observed in the LECN of our college BDs is in accordance with the findings reported by Jansen et al. (2015), Krmpotich et al. (2013) and Zhu et al. (2015), who also noticed increased intrinsic rsFC in the LECN of alcoholics and other drug-addicted individuals. Specifically, Jansen et al. (2015) and Zhu et al. (2015) described within-network alterations only in the LECN, and no changes in the RECN. The authors attributed this lateralized increase of rsFC to a compensatory mechanism that enables the (currently sober) alcoholics to regulate/inhibit the willingness to approach rewards (i.e. alcohol) ultimately having a role in lowering relapse rates (Jansen et al., 2015; Zhu et al., 2015). It seems that in order to inhibit the tendency to approach alcohol, alcoholics recruit additional resources -i.e. increased brain activity- within regions of the executive network.

Of additional interest are the reports of previously conducted morphometric and task-based fMRI studies (Doallo et al., 2014; Sousa et al., 2017; Wetherill, Squeglia, Yang, \& Tapert, 2013) that also described structural and functional abnormalities in the middle frontal gyrus of college BDs. Specifically, increased left middle frontal gyrus

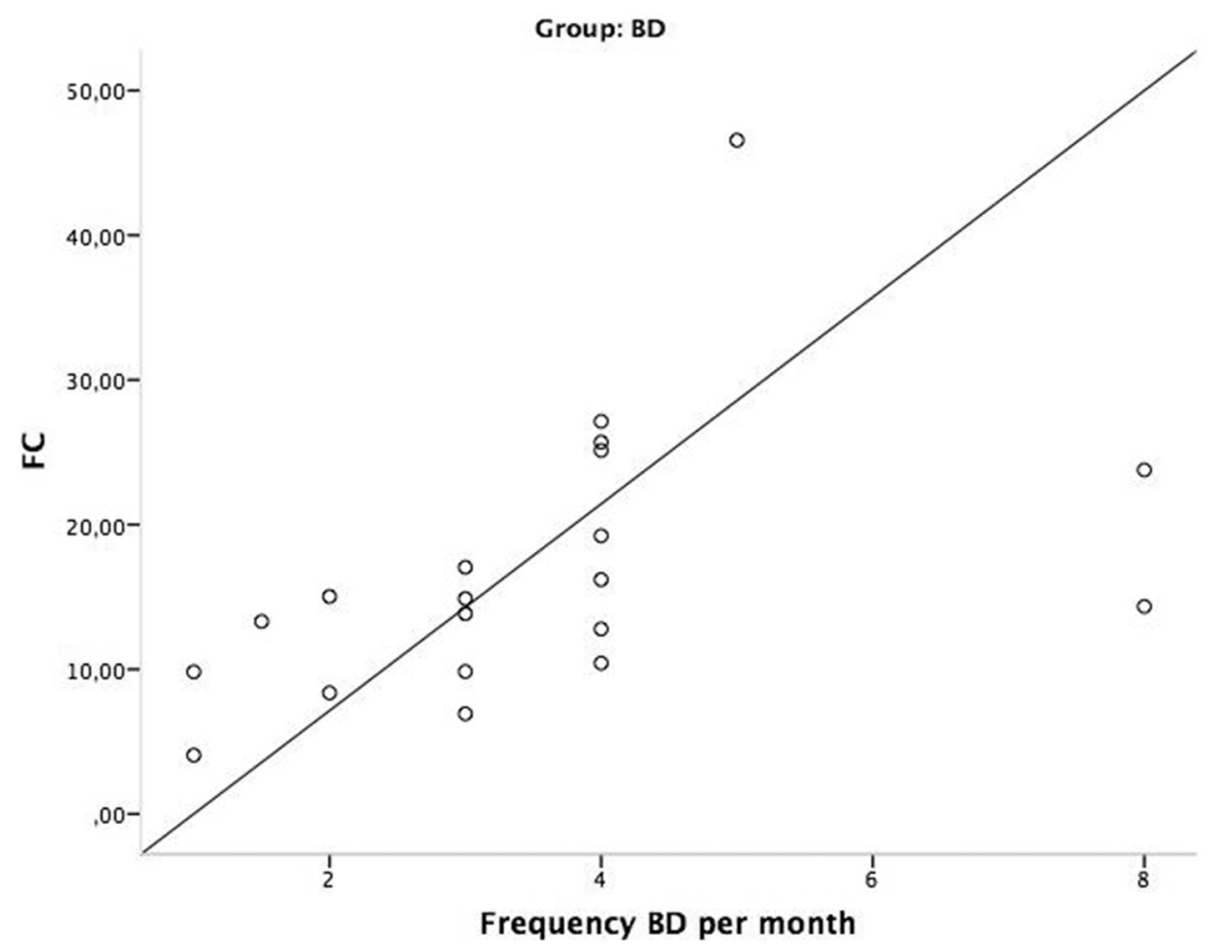

Fig. 3. Demonstrates the positive associations between the left middle frontal gyrus rsFC and the frequency of BD episodes in a month, in BDs. 
gray matter volumes were noted in college BDs (Doallo et al., 2014; Sousa et al., 2017), which were further associated with inhibitory control dysregulation (Sousa et al., 2017). In addition, increased middle frontal gyrus activation was observed in prospective BDs after transition into heavy drinking while performed a response inhibition task (Wetherill et al., 2013).

Thus, some of the studies conducted within the college BD field, using different types of neuroimaging analyses -MRI (Doallo et al., 2014; Sousa et al., 2017), fMRI (Wetherill et al., 2013) and the present resting-state fMRI study- converge in showing structural and functional alterations in the middle frontal gyrus -the prefrontal node of the LECNin college BDs. Based on these findings, we hypothesized that a disturbance of the ongoing neurodevelopmental processes as a result of the acute and intermittent alcohol ingestion during this particularly important stage of brain development might be contributing for the structural and functional impairments found in the college BDs middle frontal gyrus (for a review, see Casey et al., 2011). Contrarily, other evidence of decreased LECN functional connectivity in adult BDs was documented by Weiland et al. (2014). Besides sample composition, these inconsistent results might be associated with different fMRI pipeline analyses. Specifically, Jansen et al. (2015) and Zhu et al. (2015), who also used a data-driven independent component analysis, reported increased rsFC in the LECN of alcoholics; whereas, the network intrinsic connectivity assessment performed by Weiland et al. (2014) was based on a model-driven or seed-based analysis, which might produce some inconsistencies among studies. Particularly, while the seed-based functional connectivity is computed between the seed voxel and any other voxel in the brain, ICA decomposes BOLD signal into components or functional networks. Seed-based methods compute connectivity between two synchronous voxels irrespective of whether they belong to intrinsically connected networks; whereas ICA identifies connectivity in intrinsically connected networks and their interactions. As such, although ICA derived network maps are shown to be similar to seedbased maps, they are not identical (for a review on the relationship between both methods, see Joel, Caffo, van Zijl, \& Pekar, 2011). Nevertheless, while our goal was to address possible asynchrony just between the regions that comprise the ECN, ICA seemed the more suitable approach. Contrarily, seed-based analysis might be more appropriate for a more comprehensive evaluation of overall frontal lobe function.

Additionally, we further explored the associations between the rsFC of the left middle frontal gyrus and several alcohol-related measures. Pearson analyses revealed a positive association between the frequency of BD episodes per month and the left middle frontal gyrus rsFC. Nevertheless, this association should be interpreted with caution due to sample size limitations. The ECN is considered a central network in fundamental cognitive processes underlying goal-directed behavior, being involved in working memory, problem-solving and cognitive control (Niendam et al., 2012; Seeley et al., 2007). As such, the assessment of the ECN seems particularly relevant when addressing the neural correlates of BD since this executive network has been shown to be disrupted in multiple neurological and psychiatric disorders, including addiction (Menon, 2011; Pariyadath et al., 2016) Likewise, recent findings suggest that aberrant structure and functioning of the prefrontal node of the LECN might be a prominent feature of BD (Doallo et al., 2014; Sousa et al., 2017; Wetherill et al., 2013). In accordance, the positive association between the rsFC within the LECN and the frequency of $\mathrm{BD}$ episodes reported in the present study, suggests that acute and intermittent alcohol consumption in youth may interfere with the ECN network functioning. This disturbance eventually results in a disruption of the top-down regulation, which may lead to poor selfcontrol and diminished ability to inhibit binge alcohol consumption (Crews \& Boettiger, 2009; Crews, He, \& Hodge, 2007; Marinkovic, Rickenbacher, Azma, \& Artsy, 2012).

On the other hand, and contrary to our second hypothesis, no group differences in the left and right ECN structural connectivity were observed. Additionally, it seems that the rsFC alterations within the BDs' LECN are not being modulated by the DTI indexes (FA, AD, RD and MD). Therefore, we hypothesized that the absence of structural connectivity alterations in our BD participants might be associated with their short history of acute intermittent alcohol ingestion. Specifically, we hypothesized that the number of years of BD pattern displayed by our preclinical sample might not yet be sufficient to induce injury to white matter morphology, since these alterations have been noticed after more years of heavy drinking and higher severity of alcoholism in alcohol-dependent subjects, in an opposite way to gray matter effects as fewer years of consumption are enough to prompt alterations in this brain tissue (Fein et al., 2002; Le Berre et al., 2015; Pfefferbaum, Sullivan, Mathalon, \& Lim, 1997). Accordingly, alcohol-induced damage to the structural integrity of glial cells, including oligodendrocytes, is considered a long-term consequence of heavy alcohol consumption with significant effects on myelin sheaths and axonal integrity, as observed in the alcoholic's brain (Miguel-Hidalgo, 2018; Schulte et al., 2010). However, future studies would be of interest to further address the structural connectivity of the ECN both in BDs and AUD individuals in order to clarify this hypothesis.

Finally, we would like to acknowledge the cross-sectional nature of our study as a significant limitation when interpreting the findings, since it is not possible to unravel if the established abnormalities in the BDs' LECN are related with premorbid developmental factors or are a consequence of alcohol misuse. Other limitations include the sample size and the unbalanced sample composition as a result of the inclusion of polysubstance consumers in the BD group. Also, not having collected lifetime alcohol exposure in addition to the non-inclusion of a taskdependent paradigm are additional weaknesses of our study. These questions should be further explored in future longitudinal studies involving task-related and stimuli-independent paradigms evaluating the intrinsic connectivity of the ECN before and after alcohol initiation in youth. Moreover, streamline tractography does not account for crossing fiber regions, observed in many areas of the brain and might lead to erroneous pathway estimation (Cheng et al., 2012; Lazar and Alexander, 2003). Additional studies should perform both functional and structural connectivity analysis of the frontostriatal circuitry, which is especially relevant in addictive behaviors.

\section{Conclusion}

The findings disclosed by the analysis of this subclinical college BD group might be an important contribution to define the BD neural signatures, as they suggest that the intermittent acute alcohol consumption during this critical period -transition between adolescence and adulthood- of brain development may contribute to rsFC impairments in the ECN.

\section{Declaration of Competing Interest}

All authors declare no conflicts of interest.

\section{Acknowledgements}

This study was conducted at the Psychology Research Centre (PSI/ 01662), School of Psychology, University of Minho, and supported by the Portuguese Foundation for Science and Technology and the Portuguese Ministry of Science, Technology and Higher Education (UID/PSI/01662/2019), through the national funds (PIDDAC); and was partially supported by the project POCI-01-0145-FEDER-028672, funded by the Portuguese Foundation for Science and Technology (FCT) and the European Regional Development Fund (FEDER). SSS was supported by the SFRH/BD/88628/2012, Doctoral Fellowship of the Portuguese Foundation for Science and Technology, co-financed by POPH/FSE through QREN. Eduardo López-Caneda and Alberto Crego were supported by the FCT and the Portuguese Ministry of Science, 
Technology and Higher Education, through the national funds, within the scope of the Transitory Disposition of the Decrete No. 57/2016, of $29^{\text {th }}$ of August, amended by Law No. 57/2017 of 19 July.

\section{Appendix A. Supplementary data}

Supplementary data to this article can be found online at https:// doi.org/10.1016/j.addbeh.2019.05.033.

\section{References}

Affan, R. O., Huang, S., Cruz, S. M., Holcomb, L. A., Nguyen, E., \& Marinkovic, K. (2018). High-intensity binge drinking is associated with alterations in spontaneous neural oscillations in young adults. Alcohol, 70, 51-60. https://doi.org/10.1016/j.alcohol. 2018.01.002.

Aron, A. R., Durston, S., Eagle, D. M., Logan, G. D., Stinear, C. M., \& Stuphorn, V. (2007). Converging evidence for a fronto-basal-ganglia network for inhibitory control of action and cognition. Journal of Neuroscience, 27(44), 11860-11864.

Bari, A., \& Robbins, T. W. (2013). Inhibition and impulsivity: Behavioral and neural basis of response control. Progress in Neurobiology, 108, 44-79. https://doi.org/10.1016/j. pneurobio.2013.06.005

Bava, S., \& Tapert, S. F. (2010). Adolescent brain development and the risk for alcohol and other drug problems. Neuropsychology Review, 20(4), 398-413. https://doi.org/ 10.1007/s11065-010-9146-6.

Beckmann, C. F., \& Smith, S. M. (2004). Probabilistic independent component analysis for functional magnetic resonance imaging. IEEE Transactions on Medical Imaging, 23(2), 137-152. https://doi.org/10.1109/TMI.2003.822821.

Camchong, J., Stenger, V. A., \& Fein, G. (2013). Resting-state synchrony in short-term versus long-term abstinent alcoholics. Alcoholism, Clinical and Experimental Research, 37(5), 794-803. https://doi.org/10.1111/acer.12037.

Carbia, C., Lopez-Caneda, E., Corral, M., \& Cadaveira, F. (2018). A systematic review of neuropsychological studies involving young binge drinkers. Neuroscience and Biobehavioral Reviews, 90, 332-349. https://doi.org/10.1016/j.neubiorev.2018.04. 013.

Casey, B. J., Jones, R. M., \& Somerville, L. H. (2011). Braking and accelerating of the adolescent brain. Journal of Research on Adolescence, 21(1), 21-33.

Cheng, H., Wang, Y., Sheng, J., Sporns, O., Kronenberger, W. G., Mathews, V. P., .. Saykin, A. J. (2012). Optimization of seed density in DTI tractography for structural networks. Journal of Neuroscience Methods, 203(1), 264-272. https://doi.org/10. 1016/j.jneumeth.2011.09.021.

Correas, A., Cuesta, P., Lopez-Caneda, E., Rodriguez Holguin, S., Garcia-Moreno, L. M. Pineda-Pardo, J. A., ... Maestu, F. (2016). Functional and structural brain connectivity of young binge drinkers: A follow-up study. Scientific Reports, 6, 31293. https://doi.org/10.1038/srep31293.

Correas, A., Rodriguez Holguin, S., Cuesta, P., Lopez-Caneda, E., Garcia-Moreno, L. M. Cadaveira, F., \& Maestu, F. (2015). Exploratory analysis of Power Spectrum and functional connectivity during resting state in young binge drinkers: A MEG study. International Journal of Neural Systems, 25(3), 1550008. https://doi.org/10.1142/ S0129065715500082.

Courtney, K. E., \& Polich, J. (2009). Binge drinking in young adults: Data, definitions, and determinants. Psychological Bulletin, 135(1), 142-156. https://doi.org/10.1037/ a0014414.

Courtney, K. E., \& Polich, J. (2010). Binge drinking effects on EEG in young adult humans. International Journal of Environmental Research and Public Health, 7(5), 2325-2336. https://doi.org/10.3390/ijerph7052325.

Crane, N. A., Gorka, S. M., Phan, K. L., \& Childs, E. (2018). Amygdala-orbitofrontal functional connectivity mediates the relationship between sensation seeking and alcohol use among binge-drinking adults. Drug and Alcohol Dependence, 192, 208-214. https://doi.org/10.1016/j.drugalcdep.2018.07.044.

Crews, F., He, J., \& Hodge, C. (2007). Adolescent cortical development: A critical period of vulnerability for addiction. Pharmacology, Biochemistry, and Behavior, 86(2), 189-199. https://doi.org/10.1016/j.pbb.2006.12.001.

Crews, F. T., \& Boettiger, C. A. (2009). Impulsivity, frontal lobes and risk for addiction. Pharmacology, Biochemistry, and Behavior, 93(3), 237-247. https://doi.org/10.1016/j. pbb.2009.04.018.

Doallo, S., Cadaveira, F., Corral, M., Mota, N., Lopez-Caneda, E., \& Holguin, S. R. (2014). Larger mid-dorsolateral prefrontal gray matter volume in young binge drinkers revealed by voxel-based morphometry. PLoS One, 9(5), e96380. https://doi.org/10. 1371/journal.pone.0096380.

Dupuy, M., \& Chanraud, S. (2016). Imaging the addicted brain: Alcohol. International Review of Neurobiology, 129, 1-31. https://doi.org/10.1016/bs.irn.2016.04.003.

Fedota, J. R., \& Stein, E. A. (2015). Resting-state functional connectivity and nicotine addiction: Prospects for biomarker development. Annals of the New York Academy of Sciences, 1349, 64-82. https://doi.org/10.1111/nyas.12882.

Feil, J., Sheppard, D., Fitzgerald, P. B., Yücel, M., Lubman, D. I., \& Bradshaw, J. L. (2010). Addiction, compulsive drug seeking, and the role of frontostriatal mechanisms in regulating inhibitory control. Neuroscience \& Biobehavioral Reviews, 35(2), 248-275.

Fein, G., Sclafani, V., Cardenas, V. A., Goldmann, H., Tolou-Shams, M., \& Meyerhoff, D. J. (2002). Cortical gray matter loss in treatment-naive alcohol dependent individuals. Alcoholism: Clinical and Experimental Research, 26(4), 558-564. https://doi.org/10. 1111/j.1530-0277.2002.tb02574x.

Herman, A. M., Critchley, H. D., \& Duka, T. (2018). Binge drinking is associated with attenuated frontal and parietal activation during successful response inhibition in fearful context. The European Journal of Neuroscience. https://doi.org/10.1111/ejn. 14108.

van den Heuvel, M. P., \& Hulshoff Pol, H. E. (2010). Exploring the brain network: A review on resting-state fMRI functional connectivity. European Neuropsychopharmacology, 20(8), 519-534. https://doi.org/10.1016/j.euroneuro. 2010.03.008.

Jansen, J. M., van Wingen, G., van den Brink, W., \& Goudriaan, A. E. (2015). Resting state connectivity in alcohol dependent patients and the effect of repetitive transcranial magnetic stimulation. European Neuropsychopharmacology, 25(12), 2230-2239. https://doi.org/10.1016/j. euroneuro.2015.09.019.

Jenkinson, M., Bannister, P., Brady, M., \& Smith, S. (2002). Improved Optimization for the Robust and Accurate Linear Registration and Motion Correction of Brain Images. NeuroImage, 17(2), 825-841. https://doi.org/10.1006/nimg.2002.1132.

Joel, S. E., Caffo, B. S., van Zijl, P. C., \& Pekar, J. J. (2011). On the relationship between seed-based and ICA-based measures of functional connectivity. Magnetic Resonance in Medicine, 66(3), 644-657. https://doi.org/10.1002/mrm.22818.

Krmpotich, T. D., Tregellas, J. R., Thompson, L. L., Banich, M. T., Klenk, A. M., \& Tanabe, J. L. (2013). Resting-state activity in the left executive control network is associated with behavioral approach and is increased in substance dependence. Drug and Alcohol Dependence, 129(1-2), 1-7. https://doi.org/10.1016/j.drugalcdep.2013.01.021.

Lazar, M., \& Alexander, A. L. (2003). An error analysis of white matter tractography methods: synthetic diffusion tensor field simulations. NeuroImage, 20(2), 1140-1153. https://doi.org/10.1016/s1053-8119(03)00277-5.

Le Berre, A. P., Pitel, A. L., Chanraud, S., Beaunieux, H., Eustache, F., Martinot, J. L., .. Sullivan, E. V. (2015). Sensitive biomarkers of alcoholism's effect on brain macrostructure: Similarities and differences between France and the United States. Frontiers in Human Neuroscience, 9, 354. https://doi.org/10.3389/fnhum.2015.00354.

Lopez-Caneda, E., Cadaveira, F., Correas, A., Crego, A., Maestu, F., \& Rodriguez Holguin, S. (2017). The brain of binge drinkers at rest: Alterations in theta and Beta oscillations in first-year college students with a binge drinking pattern. Frontiers in Behavioral Neuroscience, 11, 168. https://doi.org/10.3389/fnbeh.2017.00168.

Ma, L., Steinberg, J. L., Moeller, F. G., Johns, S. E., \& Narayana, P. A. (2015). Effect of cocaine dependence on brain connections: Clinical implications. Expert Review of Neurotherapeutics, 15(11), 1307-1319. https://doi.org/10.1586/14737175.2015. 1103183.

Marinkovic, K., Rickenbacher, E., Azma, S., \& Artsy, E. (2012). Acute alcohol intoxication impairs top-down regulation of Stroop incongruity as revealed by blood oxygen leveldependent functional magnetic resonance imaging. Human Brain Mapping, 33(2), 319-333. https://doi.org/10.1002/hbm.21213.

Menon, V. (2011). Large-scale brain networks and psychopathology: A unifying triple network model. Trends in Cognitive Sciences, 15(10), 483-506. https://doi.org/10. 1016/j.tics.2011.08.003.

Miguel-Hidalgo, J. J. (2018). Molecular neuropathology of astrocytes and oligodendrocytes in alcohol use disorders. Frontiers in Molecular Neuroscience, 11, 78. https:// doi.org/10.3389/fnmol.2018.00078.

Muller-Oehring, E. M., Jung, Y. C., Pfefferbaum, A., Sullivan, E. V., \& Schulte, T. (2015) The resting brain of alcoholics. Cerebral Cortex, 25(11), 4155-4168. https://doi.org/ 10.1093/cercor/bhu134.

National Institute on Alcohol Abuse and Alcoholism [NIAAA] (2015). Alcohol facts and statistics. Retrieved from https://www.niaaa.nih.gov/alcohol-health/overviewalcohol-consumption/alcohol-facts-and-statistics.

Niendam, T. A., Laird, A. R., Ray, K. L., Dean, Y. M., Glahn, D. C., \& Carter, C. S. (2012). Meta-analytic evidence for a superordinate cognitive control network subserving diverse executive functions. Cognitive, Affective, \& Behavioral Neuroscience, 12(2), 241-268. https://doi.org/10.3758/s13415-011-0083-5.

Pariyadath, V., Gowin, J. L., \& Stein, E. A. (2016). Resting state functional connectivity analysis for addiction medicine: From individual loci to complex networks. Progress in Brain Research, 224, 155-173. https://doi.org/10.1016/bs.pbr.2015.07.015.

Pfefferbaum, A., Sullivan, E. V., Mathalon, D. H., \& Lim, K. O. (1997). Frontal lobe volume loss observed with magnetic resonance imaging in older chronic alcoholics. Alcoholism, Clinical and Experimental Research, 21(3), 521-529. https://doi.org/10. 1111/j.1530-0277.1997.tb03798.x.

Power, J. D., Barnes, K. A., Snyder, A. Z., Schlaggar, B. L., \& Petersen, S. E. (2012). Spurious but systematic correlations in functional connectivity MRI networks arise from subject motion. Neuroimage, 59(3), 2142-2154.

Schulte, T., Müller-Oehring, E. M., Rohlfing, T., Pfefferbaum, A., \& Sullivan, E. V. (2010). White matter fiber degradation attenuates hemispheric asymmetry when integrating visuomotor information. Journal of Neuroscience, 30(36), 12168-12178.

Schulte, T., Müller-Oehring, E. M., Sullivan, E. V., \& Pfefferbaum, A. (2012). Synchrony of corticostriatal-midbrain activation enables normal inhibitory control and conflict processing in recovering alcoholic men. Biological Psychiatry, 71(3), 269-278.

Seeley, W. W., Menon, V., Schatzberg, A. F., Keller, J., Glover, G. H., Kenna, H., Greicius, M. D. (2007). Dissociable intrinsic connectivity networks for salience processing and executive control. The Journal of Neuroscience, 27(9), 2349-2356. https:// doi.org/10.1523/JNEUROSCI.5587-06.2007.

Shirer, W. R., Ryali, S., Rykhlevskaia, E., Menon, V., \& Greicius, M. D. (2012). Decoding subject-driven cognitive states with whole-brain connectivity patterns. Cerebral Cortex, 22(1), 158-165. https://doi.org/10.1093/cercor/bhr099.

Smith, S. M., Fox, P. T., Miller, K. L., Glahn, D. C., Fox, P. M., Mackay, C. E., ... Beckmann, C. F. (2009). Correspondence of the brain's functional architecture during activation and rest. In: Proceedings of the National Academy of Sciences of the United States of America, 106(31), 13040-13045. https://doi.org/10.1073/pnas.0905267106.

Sousa, S. S., Sampaio, A., Marques, P., Goncalves, O. F., \& Crego, A. (2017). Gray matter abnormalities in the inhibitory circuitry of young binge drinkers: A voxel-based morphometry study. Frontiers in Psychology, 8, 1567. https://doi.org/10.3389/fpsyg 
2017.01567.

Sutherland, M. T., McHugh, M. J., Pariyadath, V., \& Stein, E. A. (2012). Resting state functional connectivity in addiction: Lessons learned and a road ahead. Neuroimage, 62(4), 2281-2295. https://doi.org/10.1016/j.neuroimage.2012.01.117.

Sutherland, M. T., Ray, K. L., Riedel, M. C., Yanes, J. A., Stein, E. A., \& Laird, A. R. (2015). Neurobiological impact of nicotinic acetylcholine receptor agonists: An activation likelihood estimation meta-analysis of pharmacologic neuroimaging studies. Biological Psychiatry, 78(10), 711-720. https://doi.org/10.1016/j.biopsych.2014.12. 021.

Taylor, P. A., Gohel, S., Di, X., Walter, M., \& Biswal, B. B. (2012). Functional covariance networks: Obtaining resting-state networks from intersubject variability. Brain Connectivity, 2(4), 203-217. https://doi.org/10.1089/brain.2012.0095.

Vergara, V. M., Liu, J., Claus, E. D., Hutchison, K., \& Calhoun, V. (2017). Alterations of resting state functional network connectivity in the brain of nicotine and alcohol users. Neuroimage, 151, 45-54. https://doi.org/10.1016/j.neuroimage.2016.11.012.
Weiland, B. J., Sabbineni, A., Calhoun, V. D., Welsh, R. C., Bryan, A. D., Jung, R. E., ... Hutchison, K. E. (2014). Reduced left executive control network functional connectivity is associated with alcohol use disorders. Alcoholism, Clinical and Experimental Research, 38(9), 2445-2453. https://doi.org/10.1111/acer.12505. Wetherill, R. R., Squeglia, L. M., Yang, T. T., \& Tapert, S. F. (2013). A longitudinal examination of adolescent response inhibition: Neural differences before and after the initiation of heavy drinking. Psychopharmacology, 230(4), 663-671. https://doi.org/ 10.1007/s00213-013-3198-2.

World Medical, A (2013). World Medical association declaration of Helsinki: Ethical principles for medical research involving human subjects. JAMA, 310(20), 2191-2194. https://doi.org/10.1001/jama.2013.281053.

Zhu, X., Cortes, C. R., Mathur, K., Tomasi, D., \& Momenan, R. (2015). Model-free functional connectivity and impulsivity correlates of alcohol dependence: A resting-state study. Addiction Biology. https://doi.org/10.1111/adb.12272. 